\title{
Meaning-based attentional guidance as a function of foveal and
}

\section{task-related cognitive loads}

\author{
Julien DAMPURE ${ }^{\mathrm{a}, \mathbf{b}}$ (corresponding author) \\ Phone: + 34 922317515, julien.dampure@ hotmail.fr
}

\section{Pedro Javier LÓPEZ-PÉREZ ${ }^{\mathrm{a}}$}

Phone: + 34 922317508, plopezpe@ull.es

\author{
Horacio A. BARBER ${ }^{\text {a,c,d }}$ \\ Phone: + 34 922317956, hbarber@ull.es
}

${ }^{a}$ Departamento de Psicología Cognitiva, Universidad de La Laguna (ULL), Tenerife, Spain.

${ }^{\mathrm{b}}$ Centre de Recherches sur la Cognition et l'Apprentissage, CNRS UMR 7295 - University of Poitiers - University of François Rabelais of Tours, Maison des Sciences de l'Homme et de la Société, Bâtiment A5, 5 rue Théodore Lefebvre, 86000 Poitiers, France.

${ }^{\mathrm{c}}$ Instituto de Tecnologías Biomédicas (ITB), Universidad de La Laguna (ULL), Tenerife, Spain.

${ }^{\mathrm{d}}$ Basque Center on Cognition, Brain and Language (BCBL), Donostia-San Sebastián, Spain.

Address for correspondence: Julien Dampuré, PhD, Department of Cognitive Psychology, Universidad de La Laguna, Campus de Guajara s/n, S/C de Tenerife, 38205, Spain.

Acknowledgments. Julien Dampuré was supported by a Postdoctoral fellowship $\left(\mathrm{N}^{\circ}\right.$ 2013.60.0019) from the Direction Générale de l'Armement (DGA, French Ministry of Defense) and supervised by Didier Bazalgette. Horacio A. Barber was supported by grant PSI2016-79624-P from the Spanish Ministry of Economy, Industry and Competitiveness.

Word count: 6877 words 


\begin{abstract}
The depth of parafoveal word processing depends on the amount of cognitive resources available. Whether this principle applies to the parafoveal semantic processing of multiple words remains, however, controversial. This study therefore aimed at testing the impact of the amount of cognitive resources available on the parafoveal semantic processing of words, by manipulating the foveal and task-related cognitive loads. Participants searched for words in displays of three semantically related or unrelated words, one of which was presented in the center of the screen and two within the parafovea. The nature of the task and the characteristics of the centered word were manipulated to vary respectively the load associated to the task and to the foveal load. Analyses revealed more first saccades toward the parafoveal semantic distractors when both loads were low. These results indicate that fast parafoveal semantic word processing is constrained by the availability of cognitive resources.
\end{abstract}

Keywords : parafoveal perception, semantic, eyetracking, visual search. 


\section{Introduction}

How visual information is selected has been extensively studied by means of the visual search paradigm, which has led to various modelizations in the context of research on attention (Wolfe, 2007). Models of visual search posit the existence of an attentional guidance mechanism based on bottom-up and top-down influences (Wolfe, 2007; Desimone \& Duncan, 1995; Deco \& Zihl, 2006), aiming at orienting attention and gaze toward relevant stimuli (Wolfe, 2007; Findlay \& Gilchrist, 2005; Zelinsky, 2008). Top-down attentional orientation (Downing, 2000; Olivers, Meijer, \& Theeuswes, 2006; Soto \& Humphreys, 2007; Soto, Heinke, Humphreys, \& Blanco, 2005) is based on involuntary processes that bias competition between visual stimuli (Desimone \& Duncan, 1995). These mechanisms rely on a comparison between the parallel processing of stimuli in the visual field and the representations of the target (i.e. target-template) maintained in working memory. Although it has been traditionally considered that only the perceptual characteristics of the stimuli can guide attention (Wolfe \& Horowitz, Kenner, Hyle, \& Vasan, 2004), some studies of visual search for objects have shown that semantic representations can also be accessed to orient individuals' behaviour, as attested by spontaneous saccades toward objects semantically related to targets, particularly in target-absent trials (Moores Laiti, \& Chelazzi, 2003; Telling, Kumar, Meyer, \& Humphreys, 2010; Huettig \& McQueen, 2007; Meyer, Belke, Telling, \& Humphreys, 2007). Moores et al. (2003) explained this phenomenon in regard to semantic priming, i.e. the semantic associates of the target are activated by spreading activation when the target-template is implemented in Working Memory (WM). Consequently, semantic associates would have an advantage in the competition, compared to objects unrelated to the target (see also, Huettig \& McQueen, 2007; Telling et al., 2010).

Despite the extension of the models of visual search to words has been questioned (for extended discussions see Léger, Ros, Rouet, \& Vibert, 2012; Dampuré, Ros, Rouet, \& Vibert, 2014), visual search for verbal material has been far less studied even though it is one 
of the most practiced activity on the web (Hsieh-Yee, 2001) and would be even more practiced than reading for comprehension (Rouet, 2006). Consequently, few is known in this activity about the attentional guidance mechanisms and the corresponding (semantic) processing of words locating in parafovea, i.e. the part of the visual field surrounding the fovea, extending from 2 to 5 degrees of visual angle which is particularly involved in information pre-processing, attention guidance and saccade programming (Rayner, 2009; Wolfe, 2007; Findlay \& Gilchrist, 2005). In this article, we purpose that meaning-based attentional guidance depends on the amount of cognitive resources available according to the difficulty of the task and of the concomitant foveal processing. To date and to the best of our knowledge, no study has directly examined the simultaneous impact of both foveal and taskrelated cognitive loads on meaning-based attentional guidance. Since contrary to a pictorial stimulus, a word has an orthographic code (i.e., visual representation) conventionally associated to a high-level, abstract mental representation (i.e., meaning), this study directly tackle the extent to which the cognitive system is able to quickly access and use the meaning of words presented in parafoveal vision. Knowing more about the impact of these factors on the attentional guidance mechanism and how they interact is thus an essential step in the understanding of complex tasks such as visual search or reading.

This question has mostly been studied in the context of reading activity. Though it has often been concluded that in sentence reading, parafoveal word processing was restricted to (sub)-lexical representations (i.e. physical appearance, orthography, phonology) and not be fast enough to access word meanings (for review, Rayner, White, Kambe, Miller, \& Liversedge, 2003; Rayner, Schotter \& Drieghe, 2014; Lee, Legge \& Ortiz, 2003), recent behavioural, eyetracking and Event Related Potential (ERP) studies in Chinese (e.g. Yeh, He, \& Cavanagh, 2012; Li, Niefind, Wang, Sommer, \& Dimigen, 2015; Zhang, Li, Wang, \& Wang, 2015; Yan, Richter, Shu, and Kliegl, 2009) and in occidental writing systems (e.g. López-Peréz, Dampuré, Hernández-Cabrera, \& Barber, 2016; Hohenstein \& Kliegl, 2014; 
Schotter, 2013; Hohenstein, Laubrock, \& Kliegl, 2010) have demonstrated that the meaning of words in parafoveal vision could be processed. The extent to which the meaning of parafoveal words can be processed during reading depends on several factors such as the depth of orthography of the language (Hohenstein, Laubrock, \& Kliegl, 2010; Yan, Richter, Shu, \& Kliegl, 2009; Yan, Zhou, Shu, \& Kliegl, 2012), the capitalization of the words' first letters (Rayner, Schotter \& Drieghe, 2014; Schotter, 2013; but see Hohenstein \& Kliegl, 2014), the degree of similarity between preview and target, but also on the expectancy of the word in parafovea (Barber, van der Meij, \& Kutas, 2013; Stites, Payne, \& Federmeier, 2017; Barber, Doñamayor, Kutas, \& Münte, 2010) and attentional resources available. Accordingly, Payne, Stites, and Federmeier (2016) recently demonstrated that the depth of parafoveal word processing during reading depended on the amount of cognitive resources needed to process the foveal word (see also Veldre \& Andrews, 2015).

Similar controversy has been formulated in the context of visual search for words, with some eyetracking studies reporting a lack of parafoveal semantic effects (e.g. Dimigen, Kliegl, \& Sommer, 2012; Léger, Rouet, Ros, \& Vibert, 2012), and one recent study showing semantic effects on parafoveal word processing, depending on the difficulty of the task (Dampuré, Ros, Rouet, \& Vibert, 2014). More precisely in this latter study, participants searched for a target word either given in advance before each trial (i.e. literal task, e.g. search for "raven") or defined by its semantic category (i.e. categorical task, e.g. search for a type of bird), among semantically related and semantically unrelated distractor words. Results showed that semantic distractors were spontaneously gazed at more often in the literal task than in the categorical task. Thus, increasing the task demands reduces the amount of cognitive resources devoted to parafoveal processing and attentional guidance toward target features (Lavie \& De Fockert, 2005). Soto and Humphreys (2008) have indeed demonstrated that increasing WM load (Experiment $3 \& 4$ ) and/or imposing an articulatory suppression task (Experiment $2 \& 4$ ) decreases the quality of the top-down attentional guidance in visual 
search for objects. In the context of the Dampuré et al. study, task-related effects on meaningbased attention guidance may be related to the precision of the target-template (i.e. the representation of the target), according to the task (Schmidt, MacNamara, Proudfit, \& Zelinsky, 2014). Indeed, the target-template formed during a categorical task is considered as broader and less well-defined than a target-template created during a literal task (Hout \& Goldinger, 2015) as it integrates multiple possible candidates and associated, but irrelevant, representations. As a consequence, (1) the maintenance in WM of the target-template in the categorical task would be resource-consuming (Schmidt et al., 2014) and (2) target-related (e.g. semantic) representations may receive less (pre)activation in a categorical task than in a literal task and thus less power in the competition with irrelevant stimuli for attentional guidance (Wolfe, 2007, Desimone \& Duncan, 1995).

In addition to task-related cognitive load, the difficulty of foveal processing has been shown to modulate the amount of cognitive resources that can be devoted to parafoveal processing. Indeed, in addition to the attentional guidance mechanisms which determines the order in which the stimuli are examined in the visual field, visual search models posit the existence of a late recognition mechanisms which aims at categorizing the item that is gazed as being the target or a distractor (Zelinsky, 2008; Findlay \& Gilchrist, 2005; Wolfe, 2007; Desimone \& Duncan, 1995; Deco \& Zihl, 2006). Since both mechanisms are closely related and resource-dependent, an increase of the foveal processing difficulty (i.e. recognition mechanism) would hinder the processing of information located in parafoveal vision (i.e. attentional guidance mechanisms). This has been referred as the tunnel vision effect in the attention research (Williams, 1988) and as the foveal load effect in the literature on reading. In the latter, for instance, Henderson and Ferreira (1990) and Schroyens, Vitu, Brysbaert and d'Ydewalle (1999) demonstrated that the preview benefit, that is the facilitation in processing the upcoming word during reading, was greater when the word located in foveal vision was frequent in the language than when it was infrequent (see also Payne, Stites, \& Federmeier, 
2016). Similarly in the context of visual search for words, Dampuré et al. (2014) reported some post-hoc evidence for a possible impact of foveal load on attentional guidance. More precisely in the categorical task (i.e. high task-related cognitive load), semantic distractors attracted the gaze in the categorical task only at the very beginning of the search when participants did not gaze directly at a word. However in the literal task (i.e. low task-related cognitive load), semantic distractors attracted the gaze also when participants were previously gazing at a word, but only if it was another semantic distractor. Altogether, and in addition to the lexical frequency, the semantic relatedness of the words seems to differentially modulate the foveal load according to the search task. Because the decision criterion is perceptuallybased in the literal task, the semantic properties of the foveal word facilitated and biased the processing toward the parafoveal semantic distractor. In the categorical task, gazing at a word increases the foveal load since each word has to be semantically processed, particularly when gazing at a semantic distractor since it needs more efforts to be rejected due to its semantic similarity with the target.

Despite these pieces of evidence, to date and to the best of our knowledge, no study has directly examined the impact of both foveal and task-related cognitive loads on meaningbased attentional guidance. Therefore, the present study aimed at testing the simultaneous impact of both foveal and task-related cognitive loads on parafoveal word semantic processing. We expected that the lower the cognitive load, the more efficient the parafoveal word processing. Participants searched for target words within successive three-word displays composed of one word at the center of the screen and two lateralized words located within participants' inferior parafoveal visual field. In target-absent displays, one of the two lateralized words was semantically related with the target word. As in previous studies of visual search for objects (e.g. Moores et al., 2003), we expected semantic attentional guidance to be reflected by spontaneous saccades toward parafoveal semantic distractors. We manipulated the task-related cognitive load by varying the nature of the visual search task 
(Dampuré et al., 2014; Dampuré et al., 2016; Schmidt et al., 2014), which was either a literal (low task-related cognitive load) or a categorical (high task-related cognitive load) search task. The semantic relationship with the target word and the lexical frequency of the centered word were manipulated to vary the foveal load. The word processing literature has indeed consistently demonstrated that lexical frequency is a strong determinant of foveal processing difficulty. Accordingly, we expected that, independently of the nature of the search task, the foveal load should be greater when gazing at an infrequent word compared to a frequent word (Henderson \& Ferreira, 1990; Schroyens et al., 1999). In addition, previous studies (Dampuré et al., 2014; Dampuré et al., 2016; Léger et al., 2012) have demonstrated that semantic distractors facilitate word processing in the literal task and generate interference in the categorical task. Taken together, we expected more first saccades toward parafoveal semantic distractors (1) in the literal task (low task-related cognitive load) when gazing at a frequent, semantically related word (low foveal load), and (2) in the categorical task (high task-related cognitive load) when gazing at a frequent unrelated distractor (low foveal load), although this latter effect should be weaker due to the higher task-related cognitive load. Finally, analyses were conducted on gaze durations on the centered words before triggering the first saccade in order to have a complete picture of the processing at play. Gaze durations were expected to be shorter in the literal task compared to the categorical task (Dampuré et al., 2014) and longer in the low, compared to the high foveal load condition (Schroyens et al., 1999). Whether gaze durations could differ according to the destination of the first saccade (semantically related vs. unrelated distractor) was particularly examined.

\section{Methods}

\section{Participants}

Seventy students (53 women) from the University of La Laguna participated in this study. They were all Spanish native speakers, right-handed, aged from 18 to 33 years old (M 
$=23$ y.o., $\mathrm{SD}=4$ ), with normal or corrected-to-normal vision and gave their written consent prior to the experiment. This study was approved by the Ethical Review Committee of the University of La Laguna.

\section{Apparatus}

Stimuli were displayed by the E-Prime 2.0 software on a 17 -inch monitor (resolution 768 x 1024 pixels). Eye movements were collected using an EyeLink 1000 recording at a $1000 \mathrm{~Hz}$ sampling rate, and preprocessed via its dedicated software DataViewer (SR Research). Participants sat $70 \mathrm{~cm}$ from the screen with their heads resting on a chinrest adjusted to a comfortable position. Because the fovea covers about $2^{\circ}$ of visual angle (Jocob \& Hochstein, 2009), the foveal vision corresponded to a circle with a diameter of $24 \mathrm{~mm}$ on screen.

\section{Material}

The study consisted of two experiments of visual search for words (see Figure 1). The words used in this study were all Spanish nouns of 6 letters length on average (from 4 to 9), written in white on a black background in 20-point Calibri font. Each word measured $5 \mathrm{~mm}$ height on screen and covered about $0.51^{\circ}$ of visual angle.

This first manipulation aimed at varying the cognitive load by manipulating the nature of the search task (i.e. factor "Task", see Figure 1) according to the instructions given at the beginning of the experiment. Specifically, in the literal task, each trial began with the presentation of two target words belonging to the same superordinate category (e.g. search for pigeon and blackbird), whereas in the categorical task, although the same target words were used, only their superordinate semantic category was given in instructions to participants (e.g. search for "types of birds"). The two tasks were performed by two different pools of 
participants but with the same 32 search trials, which consisted in searching for the target words in successive visual search displays.

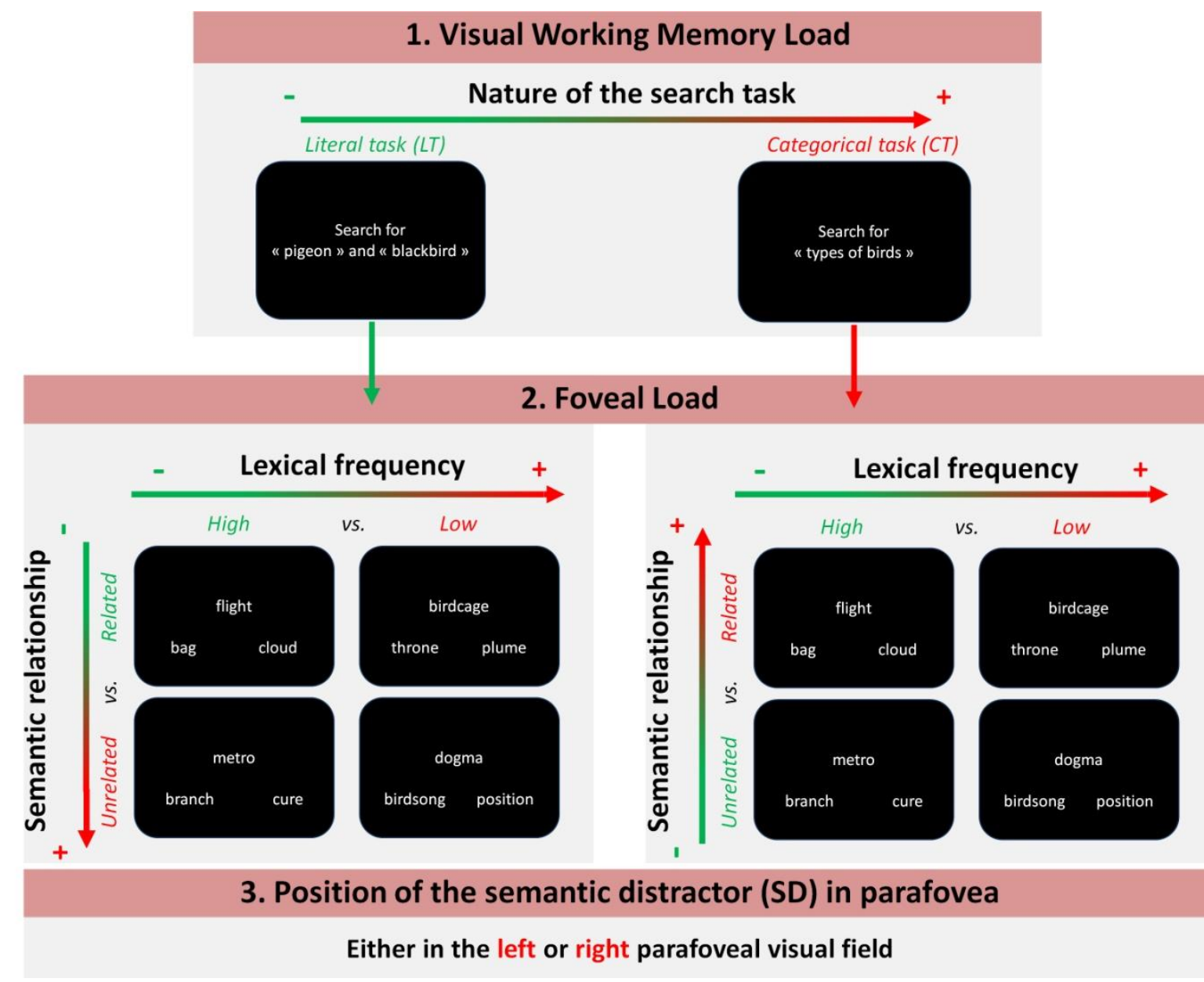

Figure 1. Presentation and illustration of the three independent variables of the study, namely: the (1) Task-related cognitive load resulting from the manipulation of the nature of the visual search tasks (literal task vs. categorical task), (2) Foveal Load deriving from the manipulation of both Lexical frequency (high vs. low) and Semantic relationship (related vs. unrelated) of the centered word with the target words, and (3) Position of the semantic distractor in participant's parafoveal visual field (left vs. right). Colored arrows (from low load in green to high load in red) and plus/minus symbols indicate the hypothesized variations of cognitive load associated to the experimental manipulations. 
The number of three-word displays varied from 7 to 23 for a given search trial, limiting the possibility of predicting the occurrence of the target words. Each of the threeword displays was made up of one word at the center of the screen (see Figure 2, Panel A), and two words in left inferior and right inferior parafoveal visual fields at $5^{\circ}$ of visual angle from the centered word (center-to-center). Choice was made to present parafoveal words into the inferior visual field since it produces less variation in retinal eccentricity of the letters compared to when words are presented into the left or right visual fields (Lee, Legge \& Ortiz, 2003). In other words, this would limit the effect of crowding due to the lateralization of words. Besides, this is why presenting words in the lower visual field rather than in the upper visual field has been consider as a better option for reading in the context of clinical studies (for review, see Chung, 2010; Petre, Hazel, Fine, \& Rubin, 2000; Chung, Mansfield, \& Legge, 1998).

For each display, participants were required to report the presence or absence of a target word. In total, over the 32 search trials, participants saw 352 three-word displays of which 152 displayed a target word (i.e. target-present displays). As in Dampuré et al. (2016), for each search trial, one of the two target words only appeared once and always in the last three-word display. The other target word was repeated at different locations in several threeword displays during a given search trial. Over the 32 search trials, the repeated target word appeared 76 times in the center of the screen, 38 in the left parafoveal visual field and 38 in the right parafoveal visual field. These manipulations were undertaken in order to (1) encourage participants to pay attention to every part of each three-word display (even the center of the screen) until the end of each search trial (i.e. to search for the final target), and (2) to limit the repetition of "no" responses participants had to make in the search displays containing only distractors, since the participants were instructed to press the button referring to a "yes" response to these target words. 
A.

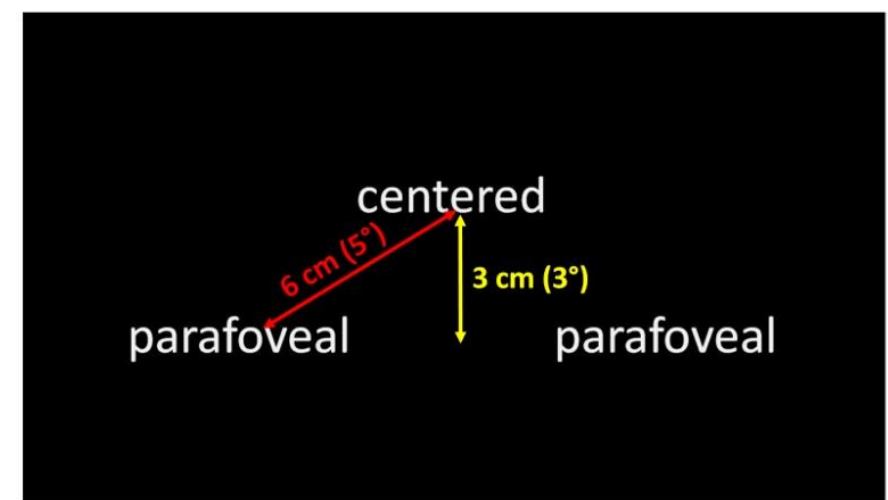

B.

Beginning of a Trial

- Literal task: memorize the two targets

- Categorical task: memorize the category of the targets

Fixation cross

Gaze at the cross without blinking

\section{Three-words display}

Is the target you are looking for present (yes/no)?

End of a Trial

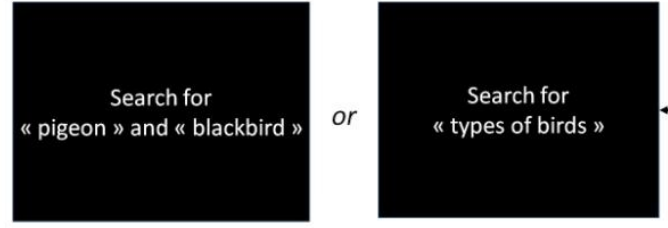

Until button press

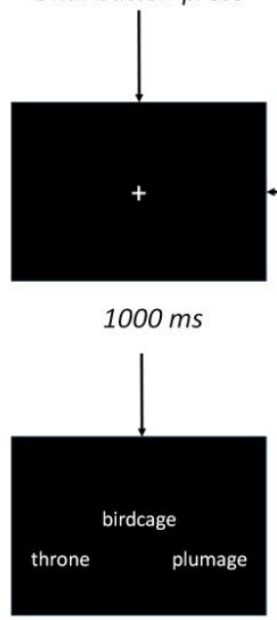

Until button press

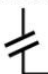

Figure 2. Panel A presents an experimental three-word display and the distances between words (both in centimetres and degree of visual angle). Panel B is the schematic representation of a search trial. The right side of the figure presents the procedure used to present the probe (i.e. the target words or their category) and each of the associated threeword displays. The left side of the figure displays, for each stage of the procedure, the corresponding instructions the participants were given orally during the training session. 
The 200 remaining three-word displays (i.e. target-absent displays) were used to test parafoveal semantic processing by manipulating the semantic relatedness of the two parafoveal words to the target (see Figure 1). One of the two parafoveal words was semantically related to the target word, while the other word was unrelated. The level of semantic association between the target word, the centered word and the parafoveal words was assessed by 133 volunteers (see Table 1). They rated on a 5-point scale different pairs of words (1: no semantic association, 5: very strong semantic association). The position of the semantically related distractor was counterbalanced so that it could appear as often within left or right inferior parafoveal visual field (i.e. factor "Position"). The two parafoveal words (i.e. semantically related and unrelated distractors) were systematically matched on their lexical frequency (systematically above 20 per million), length (about 4 to 9 letters), and number of syllables, which were assessed with the Espal database (Duchon, Perea, Sebastián-Gallés, Martí, \& Carreiras, 2013). In order to vary the foveal load, the centered word of the targetabsent displays was manipulated in terms of lexical frequency and semantic relatedness with the targets (see Figure 1). Accordingly, the centered word was either semantically related or unrelated to the target words (i.e. factor "Relatedness"), and was either common or rare in the language (i.e. factor "Frequency"), resulting in 4 modalities of 50 trials each. For example, when participants searched for the target words "paloma" and "mirlo" (pigeon and blackbird in English), they saw (1) the frequent and semantically related centered word "vuelo" (flight in English), (2) the rare and semantically related centered word "jaula" (cage in English), (3) the frequent and unrelated centered word "metro" (meter in English), and (4) the rare and unrelated centered word "dogma" (dogma in English). The length and the number of syllables were controlled so that only the lexical frequency and semantic relatedness differed between the experimental conditions (see Table 1). 
Table 1. Means of control and independent variables for centered words (Panel A), and for parafoveal words (Panel B). Parametric or non-parametric tests were conducted for each variable (only probabilities are returned).

\begin{tabular}{|c|c|c|c|c|}
\hline \multirow{3}{*}{\begin{tabular}{|ll} 
A. & \\
& \\
& Variables
\end{tabular}} & \multicolumn{3}{|c|}{ Centered-Word } & \multirow[b]{3}{*}{ Probability $(\mathrm{p})$} \\
\hline & Frequent & \multicolumn{2}{|c|}{ Infrequent } & \\
\hline & Unrelated & Related & Unrelated & \\
\hline \multirow{5}{*}{ Semantic association } & $\mathrm{M}=1,9$ & $\mathrm{M}=3,9$ & $\mathrm{M}=1,7$ & .0001 \\
\hline & \multicolumn{3}{|c|}{ Frequent, Related vs. Frequent, Unrelated } & .0001 \\
\hline & \multicolumn{3}{|c|}{ Infrequent, Related vs. Infrequent, Unrelated } & .0001 \\
\hline & \multicolumn{3}{|c|}{ Frequent, Related vs. Infrequent, Related } & .64 \\
\hline & \multicolumn{3}{|c|}{ Frequent, Unrelated vs. Infrequent, Unrelated } & .23 \\
\hline \multirow[t]{5}{*}{ Lexical frequency } & $\mathrm{M}=184,6$ & $\mathrm{M}=3,2$ & $M=3,7$ & .0001 \\
\hline & \multicolumn{3}{|c|}{ Frequent, Related vs. Frequent, Unrelated } & .52 \\
\hline & \multicolumn{3}{|c|}{ Infrequent, Related vs. Infrequent, Unrelated } & .99 \\
\hline & \multicolumn{3}{|c|}{ Frequent, Related vs. Infrequent, Related } & .0001 \\
\hline & \multicolumn{3}{|c|}{ Frequent, Unrelated vs. Infrequent, Unrelated } & .0001 \\
\hline Number of letters & $\mathrm{M}=6,2$ & $\mathrm{M}=6,6$ & $\mathrm{M}=6,3$ & .38 \\
\hline Number of syllables & $M=2,6$ & $\mathrm{M}=2,7$ & $\mathrm{M}=2,8$ & .99 \\
\hline B. & \multicolumn{3}{|c|}{ Parafoveal-Words } & \\
\hline Variables & Related & & ated & Probability $(\mathrm{p})$ \\
\hline Semantic association & $\mathrm{M}=4,1$ & \multicolumn{2}{|c|}{$\mathrm{M}=1,9$} & .0001 \\
\hline Lexical frequency & $M=69,9$ & \multicolumn{2}{|c|}{$M=65,5$} & .54 \\
\hline Number of letters & $\mathrm{M}=6,1$ & \multicolumn{2}{|c|}{$\mathrm{M}=6,1$} & 1 \\
\hline Number of syllables & $\mathrm{M}=2,58$ & \multicolumn{2}{|c|}{$\mathrm{M}=2,59$} & 1 \\
\hline
\end{tabular}




\section{Procedure}

All participants were tested individually. After a calibration, they carried out a 10 minute training session in which they received orally all the instructions necessary to perform the task (see Figure 2, Panel B). Once familiarized with the task requirements, they engaged in the experimental session for approximately 45 minutes. The target words used during the training session were different from those used during the experimental trials, were of variable lexical frequencies and were not semantically related to the target words.

Each participant performed either the literal or the categorical task. Each search trial began with the presentation of two target words (i.e. the literal task) or with the presentation of their semantic category (i.e. the categorical task). After having read this information, participants pressed a button to start the search sequence, which consisted in the presentation of successive three-word displays always preceded by a fixation cross for 1 second. During the training session, participants were instructed to always begin their search at the center of the screen, that is, to gaze first at the centered word. No further instructions concerning how to explore the display were given. Participants had to press as quickly and accurately as possible on the $\mathrm{X}$ button of a Microsoft SlideWinder joypad when they detected a target word. Otherwise, they had to press the A button. The search trial ended after all the three-word displays of the search trial had been seen by the participants.

\section{Eye movement recording and data analysis}

In accordance with the aim of the present study, all analyses were performed on the target-absent trials, that is, where a semantic distractor was presented either in left or right inferior parafoveal visual field.

Analyses of response times and error rates. The efficiency of visual search was evaluated by the error rate and the response time (in milliseconds) of the participants. Response time was defined as the time elapsed between the appearance of a three-word display and the button 
press. Response times were first submitted to a log-transformation. Then, both error rates and response times were analyzed using linear mixed models with the task (Task: literal or categorical), the semantic relationship between the centered word and the targets (Relatedness: related vs. unrelated), and the lexical frequency of the centered word (Frequency: frequent or infrequent) as fixed factors. The random structure included random slopes and intercepts of the factors Relatedness and Position (i.e. position of the parafoveal semantic distractors) for the participants, and the factor Task for the items. All mixed models were implemented in " $\mathrm{R}$ " software (version 3.4.0) by means of the ULLRToolbox (https://sites.google.com/site/ullrtoolbox/home). The logistic models were obtained using the "logit" package (Type II Wald chi-square tests), whereas the linear models were obtained with the "Ime4" package (version 1.1.13) and applied the Satterthwaite approximation for degrees of freedom (Satterthwaite, 1946).

Chi-square values $\left(\chi^{2}\right)$ and odd-ratios $(O R)$ are reported for significant effects from binomial mixed model analyses, whereas $F$ values (type III Anova) and eta-squares $\left(\eta^{2}\right)$ are reported for linear mixed model analyses.

Eye movement data analyses. Fixations were defined as any period during which the eyes remained for at least $60 \mathrm{~ms}$ within a region of $1.0^{\circ}$ of visual angle (which represented 30 pixels onscreen). Three areas of interest were drawn around each displayed word. Trials in which participants did not begin their search at screen center were excluded from the analysis ( $12 \%$ of the trials).

We first performed a binomial linear mixed model (logit) analysis on the identity of the first parafoveal word gazed at (1: semantically related, 0 : unrelated) using the task (Task: literal or categorical), the semantic relationship between the centered word and the targets (Relatedness: related or unrelated) and the lexical frequency of the centered word (Frequency: frequent or infrequent) as fixed factors. The random structure included random slopes and intercepts of the factors Relatedness and Position (i.e. position of the parafoveal semantic 
distractors) for the participants, and the factor Task for the items. Chi square values (Type II Wald chi-square tests, $\left.\chi^{2}\right)$ and odd-ratios $(O R)$ are reported.

Then, first pass gaze durations (in milliseconds) on the centered word before the first saccade triggering were analyzed by means of a linear mixed model using Task (literal or categorical), the destination of the first saccade (semantic or unrelated distractor), the Relatedness (related or unrelated) and the Frequency (frequent or infrequent) of the centered word as fixed factors. The random structure was the same as for the binomial mixed model. We report the $F$ values associated to relevant main effects and interactions and their corresponding effect sizes computed as eta-squares $\left(\eta^{2}\right)$. The $t$ values and Cohen's $d$ are reported for subsequent planned comparisons.

\section{Results}

\section{Response times and error rates}

Analysis of error rates and response times demonstrated that participants made more errors in the categorical task compared to the literal task, $\chi^{2}(1)=51.77, \mathrm{p}<.0001$, OR $=70$ (respectively, $12.3 \%$ vs. $0.8 \%$ ), and had longer response times in the categorical than in the literal task, $\mathrm{F}(1,69.4)=13.92, \mathrm{p}<.0004, \eta^{2}=.62$ (respectively, $1606 \mathrm{~ms}$ vs. $\left.1266 \mathrm{~ms}\right)$. In addition, participants made more errors when the centered word was semantically related to the target word compared to when it was unrelated, $\chi^{2}(1)=12.96, p<.0003$, OR $=1.14$ (respectively, $8 \%$ vs. $5 \%$ ). However, the analysis did not reveal any main effects of the lexical frequency of the centered word on error rates, $\chi^{2}(1)=0.09, p=.76$, or on response times $\mathrm{F}(1,194)=1.08, \mathrm{p}=.30$, nor interaction between the factors. 

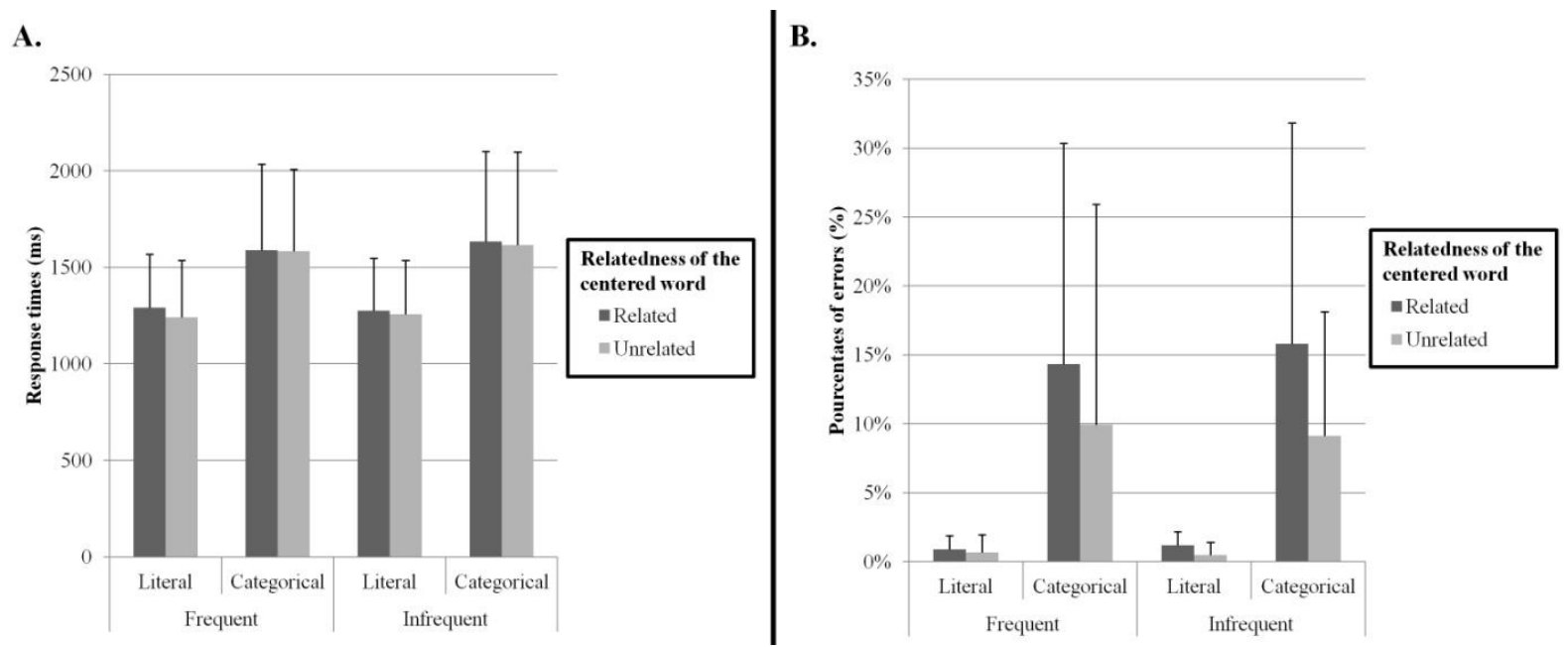

Figure 3. Means and standard deviations of response times (Panel A) and error rates (Panel B) as a function of the task (literal $v s$. categorical), the frequency (frequent $v s$. infrequent) and the semantic relationship (related vs. unrelated) of the centered word with the target words (related $v s$. unrelated).

\section{Eye movement results}

First saccade destination. The analysis performed on the direction of the first saccade (either toward a parafoveal semantic distractor or a parafoveal unrelated distractor, see Figure 4) revealed a significant interaction between the task, the relatedness of the centered word with the target and the lexical frequency of the centered word, $\chi^{2}(1)=4.06, p=.044, \mathrm{OR}=1.50$.

Follow-up analyses demonstrated that the interaction between the task and the relatedness of the centered word was significant when the centered word was frequent, $\chi^{2}(1)=$ $6.81, \mathrm{p}<.01, \mathrm{OR}=1.45$, but not infrequent, $\chi^{2}(1)=0.005, \mathrm{p}=.94$. Indeed, when the centered word was frequent and semantically related to the target, the percentage of first saccades in the direction of the parafoveal semantic distractor was higher in the literal task compared to the categorical task, $\chi^{2}(1)=6.56, \mathrm{p}=.01, \mathrm{OR}=1.29$ (respectively, $52.8 \%$ vs. $49.2 \%$ ). However, this was not true when the centered word was frequent and unrelated to the target, $\chi^{2}(1)=1.26, p=.26$ (respectively, $52 \%$ vs. $\left.49.9 \%\right)$. 


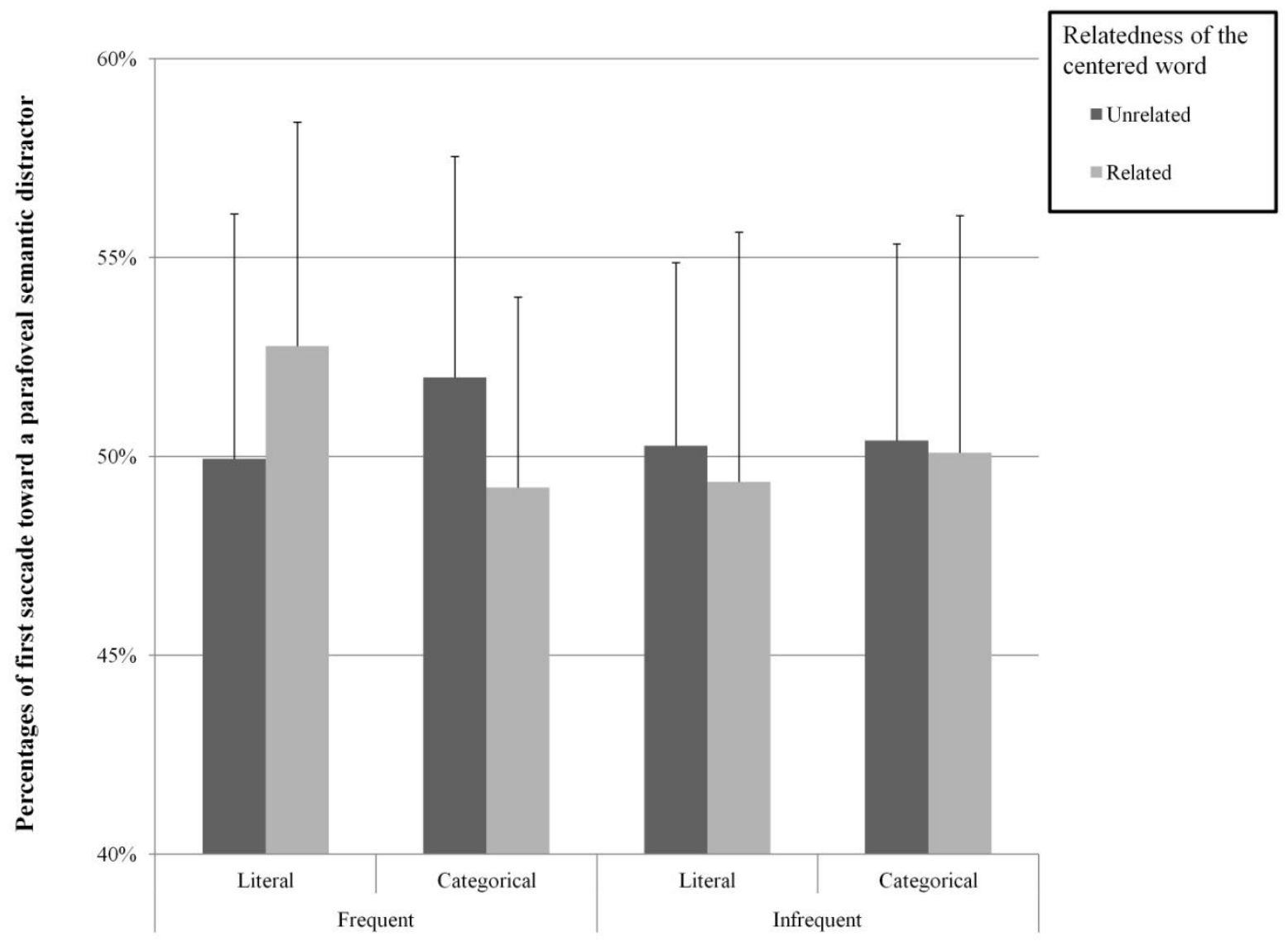

Figure 4. Percentages and standard deviations of first saccade triggered toward parafoveal semantic distractors as a function of the task (literal vs. categorical), the lexical frequency (frequent $v s$. infrequent) and the semantic relationship (related $v s$. unrelated) of the centered word with the target words (related vs. unrelated).

Gaze durations. Analyses were first conducted on the gaze durations performed on the centered word prior to the first saccade triggering and did not reveal any main effects of Task, $\mathrm{F}(1,70)=1.93, \mathrm{p}=.17$, Relatedness of the centered word, $\mathrm{F}(1,170)=0.003, \mathrm{p}=.95$, or lexical Frequency of the centered word, $\mathrm{F}(1,195)=0.99, \mathrm{p}=.32$, and were not dependent on the identity of the next word gazed at, $\mathrm{F}(1,1847)=0.87, \mathrm{p}=.35$. 


\section{Discussion}

The present study aimed at testing the impact of both foveal and task-related cognitive loads on top-down attentional guidance, more precisely on the semantic processing of parafoveal words. Specifically, the nature of the task was manipulated in order to vary the task-related cognitive load while the characteristics of the centered word (i.e. lexical frequency and semantic relatedness) were manipulated in order to modulate the foveal load. The general hypothesis was that the lower the load, the more efficient the meaning-based attentional guidance. Accordingly, results varied, depending on the task required of participants.

Literal task. The eye movement data support the existence of parafoveal semantic processing of words in the context of visual search and validate the hypothesis of an influence of the amount of cognitive resources available on attentional guidance efficiency. Indeed, in the literal task (i.e. low task-related cognitive load), first saccades were more likely to be directed toward semantic distractors when the centered word was frequent and semantically related to the target (i.e. low foveal load). Interestingly, this effect did not depend on the gaze duration made prior to triggering of the first saccade, which was insensitive to the characteristics of the centered word, and thus it cannot be attributed to an extended time for processing parafoveal words. Altogether, these results extend previous studies of visual search for objects (e.g. Moores et al., 2003) and visual search for words (Dampuré et al., 2014), suggesting that during the implementation of a low-load target-template in WM, semantic associates of targets were primed in long-term memory. The primed semantic associates would have an advantage in the top-down attentional guidance during the comparison between the targettemplate and the processing outputs of the stimuli present in the visual field (Desimone \& Duncan, 1995). However, the advantage acquired by parafoveal semantic associates for attentional guidance was cancelled when the foveal load was increased. Indeed, first saccades 
were no longer directed toward semantic distractors when the centered word was either rare in the language, or semantically unrelated to the target (Schroyens et al., 1999).

Categorical task. The task of searching for a target word defined by its semantic category was expected to increase the task-related cognitive load. Accordingly, there were more errors, longer response times and longer gaze durations in the categorical task than in the literal task. These results have previously been reported in the visual search literature (Dampuré et al., 2014; Dampuré et al., 2016; Léger et al., 2012) and emphasize the impact of the imprecision of the target-template at each stage of the visual search (Schmidt et al., 2014; Hout \& Goldinger, 2015). Accordingly, the decrease in the amount of cognitive resources available in the categorical task was expected to particularly hinder attentional guidance toward parafoveal semantic distractors. However, based on previous work (Dampuré et al., 2014), we hypothesized that parafoveal semantic distractors may attract the gaze when the centered word is a frequent unrelated distractor, a condition associated to a low foveal load in this task. Indeed in the categorical task, since distractor rejection is based on meaning, frequent unrelated distractors can be rejected faster than infrequent distractors or semantic distractors which generate interferences due to their semantic similarity with the target. Though the number of first saccades toward parafoveal semantic distractors was numerically higher than toward parafoveal unrelated distractors, the effect was not statistically significant. This might indicate that some parafoveal semantic processing occurred in the categorical task, but that access to the meaning was not fast enough to permit the cognitive system to use that information to orient the gaze toward relevant distractors. A possible explanation for this is that the level of difficulty of foveal processing in the categorical task might have increased more than expected because the searcher has to access each word meaning in order to make a decision (i.e. either to accept the word s/he is gazing at as a target or reject it as a distractor). Consequently, one could expect that when no word is presented within the fovea, that is when 
the foveal load is minimal, a parafoveal semantic distractor should be able to attract the gaze even in the categorical task. This is indeed what Dampuré et al. (2014) reported by demonstrating that parafoveal semantic distractors spontaneously attracted the gaze when participants were not gazing at another word.

Putting together the results of both tasks, the present study extends previous data that shows that even stimuli whose relationship between the visual form and the meaning is conventional can produce meaning-based attentional orienting (Dampuré et al., 2014). This emphasizes the importance for the attentional guidance mechanism of the (visual and semantic) relationship between the information in the environment and the active content of working memory, which has previously been referred to as consonance-driven orienting (Huang \& Pashler, 2008). Importantly, the present study specifies the conditions under which the attentional guidance mechanism works optimally during visual search for words by simultaneously manipulating for the first time both the foveal and task-related cognitive load. As in a previous study (Dampuré et al., 2014), we demonstrate that parafoveal semantic processing was more efficient in the literal task compared to the categorical task, that is when the task-related cognitive load is low. This effect on attentional guidance has been related to the precision of the target-template according to the task (Hout \& Goldinger, 2015; Schmidt, MacNamara, Proudfit, \& Zelinsky, 2014) whose maintenance in working memory would be resource-consuming. Critically, the present study demonstrate that the difficulty to reject the information located at fovea (i.e. the foveal load) is another source of modulation of the meaning-based attention guidance during visual search for words. In other words, meaningbased guidance depends on the interplay between an attentional guidance mechanism and a late rejection mechanism (Wolfe, 2007; Desimone \& Duncan, 1995; Deco \& Zihl, 2006; Zelinsky, 2008), with both mechanisms relying on a target-template and depending on the availability of cognitive resources. Combined with the previous data in the literature of visual 
search for words and objects, this study contribute to a unified conception of this activity, independently of the type of stimulus (e.g. objects, words, pictures).

Hence, this study specifies the conditions under which the cognitive system is able to quickly access and use the meaning of words presented in parafoveal vision and represents an essential step in the understanding of complex tasks such as visual search or even reading. Actually, we believe that the results reported in this paper may be used to extend previous work in other research areas such as reading. Indeed, the gaze durations obtained in the categorical task are very similar to those usually observed in reading (e.g. lexical frequency effects) while the parafoveal semantic processing suffered from very high variability, leading to non-significant results. Visual search and reading activities share basic principles such as parafoveal word preprocessing, relying on comparisons based on the content of working memory. In this sense, our results are consistent with those of previous reading studies that have proposed cognitive load as a critical factor to understand parafoveal word processing (e.g. Barber et al., 2013) and make a valuable contribution to the development of future general models dealing with eye movement control, which should consider the level of taskrelated cognitive load as one of the most determinant factors in foveal and parafoveal word processing. However, visual search also differs from classical reading. In reading activity, words are predictably presented according to the language writing system (from left to right in the occidental writing system). Conversely in natural condition of visual search, stimuli can appear at every single locations in the visual field and the attentional mechanisms obviously have to determine "where to go next?". In that, and even though the present study extend previous reading results in the context of clinical studies (for review, see Chung, 2010; Petre, Hazel, Fine, \& Rubin, 2000; Chung, Mansfield, \& Legge, 1998) by displaying word into the lower (left and right) visual field, future research should be conducted in order to examined the impact of the location of the visual information on parafoveal semantic processing. 


\section{References}

Barber, H. A., Doñamayor, N., Kutas, M., \& Münte, T. (2010). Parafoveal N400 effect during sentence reading. Neuroscience letters, 479(2), 152-156.

Barber, H. A., Van der Meij, M., \& Kutas, M. (2013). An electrophysiological analysis of contextual and temporal constraints on parafoveal word processing. Psychophysiology, 50(1), 48-59.

Chung, S. T. (2010). Enhancing visual performance for people with central vision loss. Optometry and vision science: official publication of the American Academy of Optometry, 87(4), 276-284.

Chung, S. T., Mansfield, J. S., \& Legge, G. E. (1998). Psychophysics of reading. XVIII. The effect of print size on reading speed in normal peripheral vision. Vision research, 38(19), 2949-2962.

Dampuré, J., Benraiss, A., \& Vibert, N. (2016). Task-dependent modulation of word processing mechanisms during modified visual search tasks. The Quarterly Journal of Experimental Psychology, 69(6), 1145-1163.

Dampuré, J., Ros, C., Rouet, J.-F., \& Vibert, N. (2014).Task-dependent sensitization of perceptual and semantic processing during visual search for words. Journal of Cognitive Psychology, 26(5), 530-549.

Deco, G., \& Zihl, J. (2006). The neurodynamics of visual search. Visual Cognition, 14(4-8), 1006-1024.

Desimone, R., \& Duncan, J. (1995). Neural mechanisms of selective visual attention. Annual Review of Neuroscience, 18, 193-222.

Dimigen, O., Kliegl, R., \& Sommer, W. (2012). Trans-saccadic parafoveal preview benefits in fluent reading: A study with fixation-related brain potentials. Neuroimage, 62(1), 381-393. 
Downing, P. E. (2000). Interactions between visual Visual Working Memory and selective attention. Psychological Science, 11(6), 467-473.

Duchon, A., Perea, M., Sebastián-Gallés, N., Martí, A., \& Carreiras, M. ( 2013 ). EsPal: Onestop Shopping for Spanish Word Properties. Behavior Research Methods, 45, 12461258.

Findlay, J. M., \& Gilchrist, I. D. (2005). Eye guidance and visual search. In G. Underwood (Ed.), Cognitive processes in eye guidance (pp. 251-281). Oxford: Oxford University Press.

Henderson, J. M., \& Ferreira, F. (1990). Effects of foveal processing difficulty on the perceptual span in reading: Implications for attention and eye movement control. Journal of Experimental Psychology: Learning, Memory, and Cognition, 16(3), 417429.

Hohenstein, S., \& Kliegl, R. (2014). Semantic preview benefit during reading. Journal of Experimental Psychology: Learning, Memory, and Cognition, 40(1), 166-190.

Hohenstein, S., Laubrock, J., \& Kliegl, R. (2010). Semantic preview benefit in eye movements during reading: A parafoveal fast-priming study. Journal of Experimental Psychology: Learning, Memory, and Cognition, 36(5), 1150-1170.

Hout, M. C., \& Goldinger, S. D. (2015). Target-templates: The precision of mental representations affects attentional guidance and decision-making in visual search. Attention, Perception, \& Psychophysics, 77(1), 128-149.

Hsieh-Yee, I. (2001). Research on Web search behavior. Library \& Information Science Research, 23(2), 167-185.

Huang, L., \& Pashler, H. (2007). Working memory and the guidance of visual attention: Consonance-driven orienting. Psychonomic Bulletin \& Review, 14(1), 148-153. 
Huettig, F., \& McQueen, J. M. (2007). The tug of war between phonological, semantic and shape information in language-mediated visual search. Journal of Memory and Language, 57(4), 460-482.

Jacob, M., \& Hochstein, S. (2009). Comparing eye movements to detected vs undetected target stimuli in an identity search task. Journal of Vision, 9(5), 20.

Lavie, N. \& De Fockert, J. (2005). The role of Visual Working Memory in attentional capture. Psychonomic Bulletin \& Review, 12(4), 669-674.

Lee, H. W., Legge, G. E., \& Ortiz, A. (2003). Is word recognition different in central and peripheral vision?. Vision Research, 43(26), 2837-2846.

Léger, L., Rouet, J.-F., Ros, C., \& Vibert, N. (2012). Orthographic versus semantic matching in visual search for words within lists. Canadian Journal of Experimental Psychology, 66, 32-43.

Li, N., Niefind, F., Wang, S., Sommer, W., \& Dimigen, O. (2015). Parafoveal processing in reading Chinese sentences: Evidence from event-related brain potentials. Psychophysiology, 52(10), 1361-1374.

López-Pérez, P. J., Dampuré, J., Hernández-Cabrera, J. A. \& Barber, H. A. (2016). Parafoveal-on-foveal and preview effects in reading: evidence from Fixation Related Potentials. Brain and Language, 162, 29-34.

Meyer, A. S., Belke, E., Telling, A. L., \& Humphreys, G. W. (2007). Early activation of object names in visual search. Psychonomic Bulletin \& Review, 14(4), 710-716.

Moores, E., Laiti, L., \& Chelazzi, L. (2003). Associative knowledge controls deployment of visual selective attention. Nature Neuroscience, 6(2), 182-189.

Olivers, C. N, Meijer, F, \& Theeuwes, J. (2006). Feature-based memory-driven attentional capture: Visual working memory content affects visual attention. Journal of Experimental Psychology: Human Perception and Performance, 32, 1243. 
Payne, B. R., Stites, M. C., \& Federmeier, K. D. (2016). Out of the corner of my eye: Foveal semantic load modulates parafoveal processing in reading. Journal of Experimental Psychology: Human Perception and Performance, 42(11), 1839-1857.

Petre, K. L., Hazel, C. A., Fine, E. M., \& Rubin, G. S. (2000). Reading with eccentric fixation is faster in inferior visual field than in left visual field. Optometry and Vision Science, 77(1), 34-39.

Rayner, K. (2009). Eye movements and attention in reading, scene perception and visual search. The Quarterly Journal of Experimental Psychology, 62, 1457-1506.

Rayner, K., Schotter, E.R., \& Drieghe, D. (2014). Lack of parafoveal semantic preview benefit in reading revisited. Psychonomic Bulletin \& Review, 21, 1067-1072.

Rayner, K., White, S.J., Kambe, G., Miller, B., \& Liversedge, S. (2003). On the processing of meaning from parafoveal vision during eye fixations in reading. In J. Hyönä, R. Radach, \& H. Deubel (Eds.). The Mind's Eye: Cognitive and Applied Aspects of Eye Movement Research (pp. 213-234). Amsterdam: Elsevier.

Rouet, J.-F. (2006). The Skills of Document Use: From Text Comprehension to Web-Based Learning. Mahwah, NJ : L. Erlbaum Associates.

Satterthwaite, F. E. (1946). An approximate distribution of estimates of variance components. Biometrics bulletin, 2(6), 110-114.

Schmidt, J., MacNamara, A., Proudfit, G. H., \& Zelinsky, G. J. (2014). More target features in visual working memory leads to poorer search guidance: Evidence from contralateral delay activity. Journal of vision, 14(3), 8-8.

Schotter, E. R. (2013). Synonyms provide semantic preview benefit in English. Journal of Memory and Language, 69(4), 619-633. 
Schroyens, W., Vitu, F., Brysbaert, M., \& d'Ydewalle, G. (1999). Eye movement control during reading: Foveal load and parafoveal processing. The Quarterly Journal of Experimental Psychology: Section A, 52(4), 1021-1046.

Soto, D., Heinke, D., Humphreys, G. W., \& Blanco, M. J. (2005). Early, involuntary topdown guidance of attention from working memory. Journal of Experimental Psychology: Human Perception and Performance, 31, 248-261.

Soto, D., \& Humphreys, G. W. (2007). Automatic guidance of visual attention from verbal working memory. Journal of Experimental Psychology: Human Perception and Performance, 33(3), 730-737.

Soto, D., \& Humphreys, G. W. (2008). Stressing the mind: The effect of cognitive load and articulatory suppression on attentional guidance from working memory. Attention, Perception, \& Psychophysics, 70(5), 924-934.

Stites, M. C., Payne, B. R., \& Federmeier, K. D. (2017). Getting ahead of yourself: Parafoveal word expectancy modulates the N400 during sentence reading. Cognitive, Affective, \& Behavioral Neuroscience, 17(3), 475-490.

Telling, A. L., Kumar, S., Meyer, A. S., \& Humphreys, G. W. (2010). Electrophysiological evidence of semantic interference in visual search. Journal of Cognitive Neuroscience, 22(10), 2212-2225.

Veldre, A., \& Andrews, S. (2015). Parafoveal preview benefit is modulated by the precision of skilled readers' lexical representations. Journal of Experimental Psychology: Human Perception and Performance, 41(1), 219.

Williams, L. J. (1988). Tunnel vision or general interference? Cognitive load and attentional bias are both important. The American Journal of Psychology, 101(2), 171-191. 
Wolfe, J. M. (2007). Guided Search 4.0: Current Progress with a model of visual search. In W. Gray (Ed.), Integrated Models of Cognitive Systems (pp. 99-119). New York: Oxford University Press.

Wolfe, J. M., Horowitz, T. S., Kenner, N., Hyle, M., \& Vasan, N. (2004). How fast can you change your mind? The speed of top-down guidance in visual search. Vision Research, 44(12), 1411-1426.

Yan, M., Richter, E. M., Shu, H., \& Kliegl, R. (2009). Readers of Chinese extract semantic information from parafoveal words. Psychonomic bulletin \& review, 16(3), 561-566.

Yan, M., Zhou, W., Shu, H., \& Kliegl, R. (2012). Lexical and sublexical semantic preview benefits in Chinese reading. Journal of Experimental Psychology: Learning, Memory, and Cognition, 38(4), 1069.

Yeh, S. L., He, S., \& Cavanagh, P. (2012). Semantic priming from crowded words. Psychological science, 23(6), 608-616.

Zelinsky, G. J. (2008). A theory of eye movements during target acquisition. Psychological Review, 115, 787-835.

Zhang, W., Li, N., Wang, X., \& Wang, S. (2015). Integration of Sentence-Level Semantic Information in Parafovea: Evidence from the RSVP-Flanker Paradigm. PloS one, 10(9), e0139016. 